\title{
150 million years of sustained increase in pterosaur flight efficiency
}

Article

Accepted Version

Venditti, C. ORCID: https://orcid.org/0000-0002-6776-2355, Baker, J. ORCID: https://orcid.org/0000-0003-4904-6934, Benton, M. J. ORCID: https://orcid.org/0000-0002-4323-1824, Meade, A. and Humphries, S. ORCID: https://orcid.org/00000001-9766-6404 (2020) 150 million years of sustained increase in pterosaur flight efficiency. Nature, 587. pp. 83-86. ISSN 0028-0836 doi: https://doi.org/10.1038/s41586-0202858-8 Available at https://centaur.reading.ac.uk/93748/

It is advisable to refer to the publisher's version if you intend to cite from the work. See Guidance on citing.

Published version at: http://dx.doi.org/10.1038/s41586-020-2858-8

To link to this article DOI: http://dx.doi.org/10.1038/s41586-020-2858-8

Publisher: Nature Publishing Group

All outputs in CentAUR are protected by Intellectual Property Rights law, including copyright law. Copyright and IPR is retained by the creators or other copyright holders. Terms and conditions for use of this material are defined in the End User Agreement.

\section{www.reading.ac.uk/centaur}




\section{CentAUR}

Central Archive at the University of Reading

Reading's research outputs online 
$1 \mathbf{1 5 0}$ million years of sustained increase in pterosaur flight efficiency

2 Chris Venditti ${ }^{1 *}$, Joanna Baker ${ }^{1}$, Michael J. Benton ${ }^{2}$, Andrew Meade ${ }^{1}$ and Stuart

3 Humphries $^{3^{*}}$

4 Affiliations:

$5{ }^{1}$ School of Biological Sciences, University of Reading, Reading RG6 6BX, United

6 Kingdom.

$7 \quad{ }^{2}$ School of Earth Sciences, University of Bristol, Life Sciences Building, Tyndall

8 Avenue, Bristol BS8 1TQ, United Kingdom.

$9 \quad{ }^{3}$ School of Life Sciences, University of Lincoln, Joseph Banks Laboratories, Green

10 Lane, Lincoln LN6 7DL, United Kingdom.

*Correspondence to c.d.venditti@reading.ac.uk and shumphries@lincoln.ac.uk.

13 Summary

14 The long-term accumulation of biodiversity has been punctuated by

15 remarkable evolutionary transitions that allowed organisms to exploit new

16 ecological opportunities. Mesozoic flying reptiles - the pterosaurs - which

17 dominated the skies for over 150 million years (myr) were the product of one such transition. The ancestors of pterosaurs were small and likely bipedal early archosaurs ${ }^{1}$, which were certainly well adapted to terrestrial locomotion.

20 Pterosaurs diverged from dinosaur ancestors in the Early Triassic ( 245 myr 21 ago, Ma), and yet their first fossils come 25 myr later, in the Late Triassic.

22 Thus, in the absence of proto-pterosaur fossils, it is difficult to study how 23 flight first evolved in this group. Our aim here is to study the evolutionary 
dynamics of pterosaurs' adaptation to a new locomotory medium. The earliest known pterosaurs took flight and subsequently appear to have become capable and efficient flyers. However, it seems clear that transitioning between forms of locomotion ${ }^{2,3}$ - from terrestrial to volant - challenged early pterosaurs by imposing a steep energetic hill to climb, thus requiring flight to provide some offsetting fitness benefits. Using novel phylogenetic statistical methods and biophysical models combined with information from the fossil record, we detect an evolutionary signal of natural selection acting to increase flight efficiency over millions of years. Our results show that there was still significant room for improvement in terms of efficiency after the appearance of flight. However, in the Azdarchoidea ${ }^{4}$, a clade exhibiting gigantism, we test the hypothesis that there was a decreased reliance on flight ${ }^{5-7}$ and find evidence for reduced selection on flight efficiency in this clade. By combining biophysical models and phylogenetic statistical methods with the fossil record, we offer a blueprint to study functional and energetic changes through geological time objectively at a far more nuanced level than has ever before been possible.

In order to determine how pterosaurs' propensity for flight changed during their evolutionary history, we calculated two indices of flight performance using a biophysical model of powered and gliding flight ${ }^{8-10}$. Firstly, we used an efficiency of flight index $\left(\mathrm{kg} \mathrm{m} \mathrm{J}^{-1}\right)$, that is the inverse of the cost of transport ${ }^{10}$, $\mathrm{CoT}^{-1}$ (see Methods and Table $\mathrm{S} 1$ for the flight model parameterization). The CoT $\left(\mathrm{J} \mathrm{kg}^{-1} \mathrm{~m}^{-1}\right)$ is the metabolic energy required to move a unit mass a unit distance at the least energetically expensive travel speed. Secondly, we calculated a sinking rate ${ }^{10}, V_{z}(\mathrm{~m}$ 
$49 \mathrm{~s}^{-1}$, see Methods) valid for gliding. A low sinking rate allows for longer travel

50 distances per glide, but also for climbing in updrafts where sinking rate must be

51 lower than the rate at which air rises from the ground. Both $\mathrm{CoT}^{-1}$ and $V_{z}$ were

52 calculated using published estimates of mass $^{11,12}$, wingspan ${ }^{13}$, wing area ${ }^{11,12}$, and

53 projected frontal area ${ }^{12}$. The dataset we use in this paper has mass and wing area

54 estimates for 16 species of pterosaur ${ }^{11}$ (Table S2, Methods). Although an alternative

55 dataset of mass and wing area estimates is available for 12 species $^{12}$, the two

56 datasets cannot be combined owing to considerable differences in the approaches of

57 each paper to body mass estimation (and the fact that the two datasets overlap).

58 However, our results are qualitatively the same using mass and wing area estimates

59 from each of the two datasets in isolation, and so here we only report the results

60 from one dataset ${ }^{11}$. We use published frontal areas $^{12}$ and wingspans ${ }^{13}$ (see Methods

61 for details).

62 Studying the changes in $\mathrm{CoT}^{-1}$ and $V_{z}$ through time can inform us about how

63 evolutionary changes such as natural selection have acted on flight performance

64 throughout the course of pterosaur evolution. The lack of proto-pterosaurs in the

65 fossil record means that it is currently impossible to be sure how the pterosaurs

66 initially overcame the energetic hill necessary to achieve flight. Our aim is to study

67 the evolutionary dynamics of pterosaurs' adaptation to a new locomotory medium.

68 The earliest known pterosaur fossils indicate they were able to fly ${ }^{14}$. If during their

69150 myr of evolution their flight performance and efficiency did not improve or

70 decrease we would expect to see no trend in $\mathrm{CoT}^{-1}$ and $V_{z}$ over time (Figure 1a). We

71 might however expect that after the start of a transition involving a change in the

72 defining medium of locomotion (i.e. from terrestrial to volant), species would be

73 relatively energetically inefficient at moving in the new environment. Thus, over time 
74 they would become more efficient. In this case, we would expect flight efficiency

$75\left(\right.$ CoT $\left.^{-1}\right)$ to increase through time and sinking rate $\left(V_{z}\right)$ to decrease (Figure $\left.1 b\right)$. We 76 would expect the opposite (a decrease in $\mathrm{CoT}^{-1}$ and an increase in $V_{z}$ over time) if 77 flight performance reduced over time (Figure 1c).

In order to study the evolution of flight (including calculation of the flight performance indices), it is necessary to account for shared ancestry owing to phylogeny. Several phylogenetic trees for pterosaurs exist in the literature e.g. 1,15,16, but none of these provides well-justified estimates of the uncertainty among species relationships and divergence dates. To account for phylogenetic and temporal uncertainty in our analyses we constructed a Bayesian dated posterior sample of phylogenetic trees for 128 pterosaurs using published character state data ${ }^{15}$ (Figure 2, Supplementary Data 1, and see Methods).

Wingspan is strongly associated with pterosaur morphologies. We find wingspan explained $97 \%$ (range of the posterior distribution, 95-98\%) of the variation in mass, $97 \%(96-98 \%)$ of the variation in wing area, and $75 \%(71-87 \%)$ of the variation in frontal area. Then using a phylogenetic prediction method ${ }^{18}$ we derived a posterior distribution of imputed masses, projected frontal area and wing area for a further 59 species of pterosaurs based on the results of the phylogenetic regression analyses and our phylogenetic tree (Table S2). Our use of Bayesian phylogenetic methods means we integrate our analyses over all phylogenetic

94 (topology and divergence dates) and model uncertainties. Thus, based on our imputations, we calculated a posterior distribution of $1000 \mathrm{CoT}^{-1}$ and $1000 \mathrm{Vz}$ estimates for use in our analyses of flight performance through time (see Methods).

97 Our final set of analyses used information from 75 species, including the uncertainty 
98 from imputed values that span the majority of the phylogenetic diversity of all known 99 pterosaurs (Figure 2a). While $\mathrm{CoT}^{-1}$ is an efficiency index related to the amount of energy needed to

101 travel a given distance, independently of how long it takes, we do expect it to 102 correlate with mass ${ }^{19}$. It is energetically cheaper for a large animal to move a given 103 mass over a particular distance than for a small animal to travel the same distance ${ }^{20}$ 104 (Figure $2 b$ ). Sinking rate is similarly affected by mass (Figure 2c) and reflects the 105 relationships we know for birds and bats (Supplementary Information). This relationship with mass means that we need to simultaneously consider

107 mass and its evolutionary association with flight performance in our analyses of flight 108 efficiency and sinking rate through time. With this in mind, pterosaurs have been reported to conform with the well-known Cope's rule ${ }^{13}$ - a phenomenon where species increase in size through geological time. The most compelling evidence for

111 this is derived from analyses reporting an increase in wingspan from $150 \mathrm{Ma}$ to the 112 end of the Cretaceous ( $66 \mathrm{Ma}$ ) coincident with the origin of birds (Avialae) $)^{13}$. 113 However, such a trend could emerge as a consequence of increased flight efficiency 114 rather than increase in body size per se. Animals with a larger wingspan for their mass are likely to be more efficient flyers ${ }^{9}$. We find using a phylogenetic regression model that accounts for the uncertainty in our inferred tree and our estimates of

117 species masses, frontal area and wing area (see Methods), that pterosaur size did 118 increase significantly through time. In addition, a model that allows the rate of mass 119 increase through time to differ before and after the origin of the birds fits significantly better than a model without such an inflection. In line with earlier conclusions ${ }^{13}$, we

121 find that there is no significant increase in size until $150 \mathrm{Ma}\left(\mathrm{p}_{\mathrm{x}}=0.59\right)$. From that 122 point the average pterosaur grew significantly from $0.60 \mathrm{~kg}$ to $6.05 \mathrm{~kg}$ (proportion of 
123 the posterior distribution that crosses zero, $p_{x}=0.02$ ), a 10-fold increase in size, over 65 million years.

Turning now to flight performance, there is a growing body of evidence indicating that the Azhdarchoidea had strong terrestrial affinities ${ }^{5-7,21}$ (cf. ${ }^{22}$ ). Here the

127 Azhdarchoidea are considered to comprise the common ancestor and all

128 descendants of Tapejara, Quetzlcoatlus, and Dsungaripterus ${ }^{1}$ (posterior nodal support $=0.83$ in our phylogeny). Evidence suggests that azhdarchoid pterosaurs had relatively inflexible necks ${ }^{7}$, left tracks indicating terrestrial proficiency ${ }^{5}$, and possessed other adaptations associated with ground-dwelling generalist foraging

132 (reviewed $\left.{ }^{6,7}\right)$. Dsungaripterids (the most basal azdharchoids in our phylogeny, Figure

133 2) are reported to have been wading foragers ${ }^{14,21}$ feeding on hard-shelled organisms 134 at water margins ${ }^{23}$. Given the terrestrial tendencies in the Azhdarchoidea compared to what we know about other pterosaurs, we might expect diminished reliance on flight, leading to the expectation that they might have differed from other pterosaurs

137 in the selection pressures for adaptations associated with flight and locomotion.

138 Thus, in our analyses we test whether the evolution of flight performance through 139 time in the Azhdarchoidea is distinct from other pterosaur species. Applying phylogenetic regression to flight efficiency through time we find that, 141 even after accounting for mass, efficiency increased significantly $\left(p_{x}=0.00\right.$, Figure $1423 a-c)$ in non-azdarchoid pterosaurs. However, in contrast to our finding for mass, 143 there is no significant effect associated with the arrival of birds $\left(p_{x}=0.47\right)$. Early 144 pterosaurs ( $<200 \mathrm{Ma}$ ) had an average efficiency of $0.29 \mathrm{~kg} \mathrm{~m} \mathrm{~J}^{-1}$ but by $70 \mathrm{Ma}$ they 145 were greater than $50 \%$ more efficient $\left(\mathrm{CoT}^{-1}=0.51 \mathrm{~kg} \mathrm{~m} \mathrm{~J}^{-1}\right)$. Congruently we find 146 that sinking rate (after accounting for mass) for non-azdarchoid pterosaurs reduced 147 from $0.80 \mathrm{~m} \mathrm{~s}^{-1}$ to $0.50 \mathrm{~m} \mathrm{~s}^{-1}$ over the course of the 150 myr of their evolutionary 
148 history (Figure 3d-f). In contrast, azdarchoids exhibited no change in efficiency or

149 sinking rate from origin to extinction.

150 Our results show that, save for azdarchoids, following their transition to volant

151 locomotion, the pterosaurs exhibited a sustained increase in flight efficiency over 150

152 myr until their extinction. To achieve this, natural selection acted to decouple the

153 evolution of body size and wingspan (Figure 3g-i) to sculpt these enigmatic

154 creatures from what might have been inefficient flyers that took to the air for only

155 short spells, to creatures that could fly long distances over extended periods. At their

156 origin, some $\sim 147 \mathrm{Ma}, 85$ myr after the origin of crown pterosaurs, azdarchoids had

157 a slightly lower flight efficiency and higher sinking rates compared with their

158 contemporaries - and showed no temporal trends in either trait until their eventual

159 extinction (Figure 3a-f). This reduced pattern of flight efficiency is also borne out in

160 analyses of gross morphology - azdarchoids arose with short wings for their size,

161 and maintained this condition until their final demise.

162 Our approach demonstrates the power of combining biophysical models and

163 phylogenetic statistical methods with the fossil record to understand the evolution of

164 flight in pterosaurs. In doing so we offer a blueprint to study functional and energetic

165 changes objectively through geological time at a far more nuanced level than has

166 ever before been possible.

\section{References}

1681 Andres, B., Clark, J. \& Xu, X. The earliest pterodactyloid and the origin of the 169 group. Curr. Biol. 24, 1011-1016, doi:http://dx.doi.org/10.1016/j.cub.2014.03.030 170 (2014). 
1712 Williams, T. M. The evolution of cost efficient swimming in marine mammals:

172 Limits to energetic optimization. Philos T R Soc B 354, 193-201 (1999).

1733 Alexander, R. M. Bioenergetics. One price to run, swim or fly? Nature 397, 174 651-653, doi:10.1038/17687 (1999).

1754 Naish, D. \& Witton, M. P. Neck biomechanics indicate that giant

176 Transylvanian azhdarchid pterosaurs were short-necked arch predators. PeerJ 5, 177 e2908 (2017).

1785 Hwang, K.-G., Huh, M., Lockley, M. G., Unwin, D. M. \& Wright, J. L. New 179 pterosaur tracks (Pteraichnidae) from the Late Cretaceous Uhangri formation, 180 southwestern Korea. Geol Mag 139, 421-435 (2002).

1816 Witton, M. P. \& Naish, D. Azhdarchid pterosaurs: Water-trawling pelican 182 mimics or "terrestrial stalkers"? Acta Palaeontol. Pol. 60, 651-660, 610 (2013).

1837 Witton, M. P. \& Naish, D. A reappraisal of azhdarchid pterosaur functional 184 morphology and paleoecology. PLOS ONE 3, e2271 (2008).

1858 Humphries, S., Bonser, R. H., Witton, M. P. \& Martill, D. M. Did pterosaurs 186 feed by skimming? Physical modelling and anatomical evaluation of an unusual 187 feeding method. PLoS Biol. 5, e204 (2007).

1889 Pennycuick, C. J. Modelling the flying bird. Vol. 5 (Elsevier, 2008).

18910 Taylor, G. \& Thomas, A. Evolutionary biomechanics: Selection, phylogeny, 190 and constraint. (Oxford University Press, 2014).

19111 Witton, M. P. A new approach to determining pterosaur body mass and its 192 implications for pterosaur flight. Zitteliana 28, 143-158 (2008). 
19312 Henderson, D. M. Pterosaur body mass estimates from three-dimensional

194 mathematical slicing. J Vert Paleontol 30, 768-785,

195 doi:10.1080/02724631003758334 (2010).

19613 Benson, R. B. J., Frigot, R. A., Goswami, A., Andres, B. \& Butler, R. J.

197 Competition and constraint drove Cope's rule in the evolution of giant flying reptiles.

198 Nat Commun 5, doi:10.1038/ncomms4567 (2014).

19914 Witton, M. P. Pterosaurs: Natural history, evolution, anatomy. (Princeton 200 University Press, 2013).

20115 Longrich, N. R., Martill, D. M. \& Andres, B. Late Maastrichtian pterosaurs from 202 North Africa and mass extinction of Pterosauria at the Cretaceous-Paleogene 203 boundary. PLoS Biol. 16, e2001663 (2018).

20416 Andres, B. \& Myers, T. S. Lone star pterosaurs. Earth Env Sci T R Soc Edinb 205 103, 383-398, doi:doi:10.1017/S1755691013000303 (2012).

20617 Schliep, K. P. Phangorn: Phylogenetic analysis in R. Bioinformatics 27, 592$207593(2011)$.

20818 Organ, C. L., Shedlock, A. M., Meade, A., Pagel, M. \& Edwards, S. V. Origin 209 of avian genome size and structure in non-avian dinosaurs. Nature 446, 180-184, 210 doi:10.1038/nature05621 (2007).

21119 Bale, R., Hao, M., Bhalla, A. P. S. \& Patankar, N. A. Energy efficiency and 212 allometry of movement of swimming and flying animals. Proc Natl Acad Sci U S A 213 111, 7517-7521, doi:10.1073/pnas.1310544111 (2014).

21420 Alexander, R. M. Models and the scaling of energy costs for locomotion. $J$ 215 Exp Biol 208, 1645-1652, doi:10.1242/jeb.01484 (2005). 
21621 Unwin, D. M. The pterosaurs from deep time. (Pi Press, New York, 2005).

21722 Averianov, A. Reconstruction of the neck of Azhdarcho lancicollis and lifestyle 218 of azhdarchids (pterosauria, azhdarchidae). Paleontol J 47, 203-209 (2013).

21923 Bestwick, J., Unwin, D. M., Butler, R. J., Henderson, D. M. \& Purnell, M. A. 220 Pterosaur dietary hypotheses: A review of ideas and approaches. Biol Rev 93, 2021$2212048(2018)$.

222

223 Figure 1: Hypothesised scenarios of the evolutionary trajectory of flight 224 performance metrics through time. a) No relationship between flight performance 225 metrics and time would indicate no directional change in flight ability through time. b) 226 An increase in efficiency through time and a reduction in sinking rate would 227 demonstrate a general tendency for selection favouring increased flight performance 228 as the pterosaurs radiated. c) A decrease in efficiency and an increase in sinking 229 rate would imply a reduction in flight performance through time. The branches of the 230 phylogenetic trees are coloured by hypothesised magnitudes of efficiency (green) 231 and sinking rate (blue).

232

233 Figure 2: Pterosaur phylogeny and the relationship of flight performance 234 metrics with mass. a) Density diagram ${ }^{17}$ showing the uncertainty in our 235 phylogenetic reconstruction for pterosaurs ( $n=128$, see Methods for details).

236 Superimposed is the maximum clade credibility tree and triangle points indicate 237 species for which we have published data on mass, wing area, frontal area, and 238 wingspan. Circle points indicate species for which we have wingspan only. Red denotes azdarchoids. b) The relationship between flight efficiency and mass $(n=75)$, 
240 with our mean phylogenetic imputations plotted with standard deviations shown by

241 the green ellipses. c) The relationship between sinking rate and mass $(n=75)$, with

242 our mean phylogenetic imputations plotted with standard deviations shown by the

243 blue ellipses.

244

245 Figure 3: Flight performance through time. Summary plots for each of our three main

246 traits of interest (efficiency, a-c; sinking rate, d-f; wingspan, g-i). A phylogeny of the species

247 included in each analysis $(\mathrm{a}, \mathrm{d}, \mathrm{g})$ is shown with branches shaded by reconstructed trait

248 values; Azdarchoidea is highlighted in red. Trait data are shown plotted against time (b, e, h)

249 where all species with imputed values are represented by circles - excepting wingspan data

250 which is all from published sources. Points are coloured by species' body mass and

251 azdarchoid species are outlined in red. The posterior distribution of model predictions for

252 each trait against time (c, f, i) shows how these traits evolved during pterosaur history.

253 These relationships demonstrate that - after accounting for size - pterosaurs continually

254 increased their efficiency (c) and wingspan (i) whilst there was a continual reduction in

255 sinking rate (f) through time (grey lines, median in black). This applies to all pterosaurs

256 except azhdarchoids (pink lines, median in red) which show no significant trends - although

257 they do have relatively short wings for their mass (i).

258

259

260

261 


\section{Methods}

263

264 All morphological data were obtained from a published phylogenetic character 265 matrix ${ }^{15}$. However, we retained only discrete morphological characters, excluding the 266

\section{Phylogenetic Inference} continuously varying characters, and treating all ordered characters as unordered, resulting in a total of 220 discrete morphological characters coded for 128 pterosaur species.

We constructed a posterior sample of time-calibrated phylogenetic trees for pterosaurs using the birth-death serial-sampling mode ${ }^{24,25}$ as implemented in BEAST v2.426 allowing for simultaneous estimation of both the topology and divergence times. For each species, we tip-dated using the midpoint of the stratigraphic age representing the first appearance of each species using published time intervals ${ }^{1,13,15}$. The origin of the birth-death process was estimated from a uniform prior distribution ranging from the age of the youngest species in the tree (Eudimorphodon rosenfeldi) up to an arbitrary upper limit of $350 \mathrm{Ma}$.

Owing to the lack of information about speciation and extinction rates in the pterosaur literature we took a conservative approach by placing a wide uninformative prior distribution (uniform ranging between 0 and infinity) on both the effective reproductive number (the birth-death ratio) and the "become uninfectious rate" (total death rate). Similarly, we placed an uninformative uniform prior between 0 and 1 on the sampling proportion. Together, these parameters enable direct estimation of birth-death rates throughout the phylogenetic tree ${ }^{24}$.

We modelled rate heterogeneity across lineages using an uncorrelated relaxed morphological clock $^{27}$. We placed an exponential prior $($ mean $=1)$ on the mean of the lognormal distribution from which the branch-wise clock rates are drawn, 
287 and a gamma prior $(\alpha=0.5396, \beta=0.3819)$ on the standard deviation. Characters 288 were partitioned on the basis of the number of discrete states, and we applied Lewis' 289 Markov k (Mk) model of morphological character evolution ${ }^{28}$ across all partitions, 290 estimating a shared gamma shape parameter $\left(\Gamma_{4}\right)^{29}$ using an exponential prior 291 distribution with mean $=1$.

292 The MCMC chain was run for one billion iterations, sampling every 100,000 293 iterations after convergence. To produce the posterior sample of 1,000 phylogenetic 294 trees used in the main analyses, we randomly sampled 1,000 iterations from this 295 chain, ensuring that all parameters had an effective sample size of $>500$, calculated 296 using Tracer v1.6 $6^{30}$. We ensured that all parameters that were estimated using a 297 uniform uninformative prior (origin, effective reproductive number, become 298 uninfectious rate, and the sampling rate) returned a posterior distribution of 299 estimates that differed from the prior. The analysis was repeated multiple times to 300 ensure convergence was reached. All chains were inspected visually using Tracer $301 \quad \mathrm{v} 1.6^{30}$

The full sample is visualized in Figure 2 a as a density tree produced in $\mathrm{R}^{31}$ using functions available in the package phangorn ${ }^{17}$, and is available to download in 304 nexus format as Supplementary Data 1 of this article.

Imputation of pterosaur measurements

To calculate our efficiency index (see below) we required mass, frontal area and

307 wing area for adult pterosaur species. Estimates for mass and wing area are 308 available for $\mathrm{N}=16$ species from Witton ${ }^{11}$ and $\mathrm{N}=12$ species from Henderson ${ }^{12}$ (see 309 Table S2). For frontal area values are taken from Henderson ${ }^{12}(\mathrm{~N}=12$, though see 310 below). Independently for each of the two datasets, we used the phylogenetic 311 method outlined in ${ }^{18}$ to impute a posterior sample of 1000 estimates of mass, frontal 
312 area and wing area for a total of $\mathrm{N}=75$ species. We obtained wingspans for

313 additional species from ${ }^{13}$ (a list of species used is found in Table S2) and used each

314 morphological trait's relationship with wingspan (i.e. a phylogenetic regression of

315 each trait against wingspan) to impute species-specific values. As a part of this

316 procedure, we also imputed frontal area for the $\mathrm{N}=7$ species in the Witton ${ }^{11}$ dataset

317 which did not have data in Henderson ${ }^{12}$ (see Table S2).

318 Flight energetics model

319 Animal powered flight energetics, while perhaps kinematically different for bats, birds

320 and pterosaurs, are still ultimately constrained by physics. It has previously been

321 demonstrated that it is possible to infer flight performance of pterosaurs using

322 biophysical models of flight in combination with metabolic scaling estimates from

323 birds $^{8}$. Here we used an actuator-disc based model owing to the pedigree of this

324 approach and because more complex wake dynamics models and computational

325 approaches are particularly difficult to parameterize, requiring a number of kinematic

326 parameters such as wingbeat frequency that are impossible to infer from fossil

327 material. We used a modified version of Pennycuick's Flight model (v1.25) ${ }^{9}$ that we

328 developed from earlier work ${ }^{8}$ and implemented in Matlab ${ }^{\circledR 32}$, and which includes

329 published parasite power estimates ${ }^{33}$. Flight performance is estimated based on

330 morphological measurements such as body mass, wingspan and wing area (Table

331 S1) and the model produces a U-shaped power-to-airspeed relationship, from which

332 a minimum power speed $\left(V_{m p}\right)$ can be calculated. This $V_{m p}$ is the least energetically

333 expensive flight speed and so provides a useful proxy for efficiency ${ }^{10}$ when

334 incorporated into the CoT.

335 We used the model to estimate the metabolic and mechanical power required 336 for powered (flapping) flight given information on a minimal set of morphological 
337 traits and estimates of physiology, as well as aerodynamic constants (Table S1). The

338 intersection of the power curve with an animal's available metabolic power ( $\mathrm{P}_{\mathrm{BMR}}$,

339 calculated from mass and estimated basal metabolic rate, BMR) allows us to

340 characterise flight ability ${ }^{9,34}$. Consistent with current thought ${ }^{35,36}$, and in line with

341 previous studies ${ }^{8}$, we assume that pterosaurs had a BMR similar to that of birds.

\section{Estimation of energetic efficiency}

343 A number of energetic efficiency measures exist ${ }^{10}$ but one useful proxy is the inverse

344 of the mass specific Cost of Transport (CoT, the energy required to move a unit 345 mass a unit distance, independent of the time taken to do so). We estimate CoT as

$346 \mathrm{PBMR} /(\mathrm{V} \times \mathrm{M})$ where $\mathrm{V}$ is the least energetically expensive travel speed (i.e. $\mathrm{V}_{\mathrm{mp}}$ ) and 347 other parameters are as in Table S1.

$348 \quad$ For species with available data (Table S2) we produced a single estimate of $349 \mathrm{CoT}^{-1}$ using the inverse of the above formula. For each of the species for which we 350 imputed mass, frontal area and wing area (Table S2 and above), we use the full 351 sample of our imputed values to produce a posterior sample of 1000 estimates of 352 CoT $^{-1}$.

As formulated, CoT accounts for mass. However, as energy efficiency appears to increase with body size ${ }^{19,20}$ we included size in our regression model of

355 CoT $^{-1}$ through time (main text and see below) to account for this.

\section{Estimation of sinking rate}

357 Sinking rate while gliding $\left(V_{z}\right)$ was calculated as $D \times V_{m p} / M \times g$ (where $D$ is the total 358 aerodynamic drag resulting from the addition of the induced, parasite and profile 359 drags) and assuming a linear wingspan reduction (see $\left.{ }^{34}\right)$. 
360 Phylogenetic regression models testing temporal trends in mass and efficiency

361 To test the evolutionary trajectories of pterosaur mass and measures of flight

362 efficiency through time we use phylogenetic generalized least squares ${ }^{37,38}$ multiple

363 regression models in a Bayesian framework. We assessed the significance of

364 regression parameters using the proportion of the posterior distribution that crosses

365 zero, $p_{x}$, where we consider $p_{x}<0.05$ as significant. In addition to the 16 species for

366 which we have single estimates of body mass, $\mathrm{Vz}$ and $\mathrm{CoT}^{-1}$ from ${ }^{11}$ (or $\mathrm{N}=12$

367 from ${ }^{12}$ ), in all our models we include the full set of posterior estimates of both body

368 mass and $\mathrm{CoT}^{-1}$ for all species for which the data are imputed (Table S2). These

369 values are sampled in proportion to their probability during the running of the MCMC

370 chain. This allows us to incorporate information about the variance of our

371 imputations, avoiding problems associated with summarizing the posterior

372 distribution into a single point estimate.

373 


\section{Methods References}

37524 Stadler, T. Sampling-through-time in birth-death trees. J. Theor. Biol. 267, $376 \quad 396-404$, doi:10.1016/j.jtbi.2010.09.010 (2010).

37725 Stadler, T., Kühnert, D., Bonhoeffer, S. \& Drummond, A. J. Birth-death 378 skyline plot reveals temporal changes of epidemic spread in HIV and hepatitis C 379 virus (HCV). Proc Natl Acad Sci U S A 110, 228-233, doi:10.1073/pnas.1207965110 380 (2013).

38126 Bouckaert, R. et al. BEAST 2: A software platform for Bayesian evolutionary 382 analysis. PLoS Comp. Biol. 10, e1003537, doi:10.1371/journal.pcbi.1003537 (2014).

38327 Drummond, A. J., Ho, S. Y. W., Phillips, M. J. \& Rambaut, A. Relaxed 384 phylogenetics and dating with confidence. PLoS Biol. 4, e88, 385 doi:10.1371/journal.pbio.0040088 (2006).

38628 Lewis, P. O. A likelihood approach to estimating phylogeny from discrete 387 morphological character data. Syst. Biol. 50, 913-925, 388 doi:10.1080/106351501753462876 (2001).

38929 Yang, Z. Maximum likelihood phylogenetic estimation from DNA sequences 390 with variable rates over sites: Approximate methods. J. Mol. Evol. 39, 306-314 391 (1994).

$39230 \quad$ Tracer v. 1. 6. 2014 (2015).

39331 R: A language and environment for statistical computing (R Foundation for 394 Statistical Computing., 2016).

39532 The Mathworks inc. (Natick, MA, 2017). 
39633 Ward, C. V. Interpreting the posture and locomotion of Australopithecus

397 afarensis: Where do we stand? Am. J. Phys. Anthropol. 119, 185-215 (2002).

39834 Pennycuick, C. J. Bird flight performance. (Oxford University Press, USA, 399 1989).

40035 Clarke, A. Dinosaur energetics: Setting the bounds on feasible physiologies 401 and ecologies. Am Nat 182, 283-297, doi:10.1086/671259 (2013).

40236 Pontzer, H., Allen, V. \& Hutchinson, J. R. Biomechanics of running indicates 403 endothermy in bipedal dinosaurs. PLoS ONE 4, e7783,

404 doi:10.1371/journal.pone.0007783 (2009).

40537 Pagel, M. Inferring evolutionary processes from phylogenies. Zool Scr 26, 406 331-348, doi:10.1111/j.1463-6409.1997.tb00423.x (1997).

40738 Freckleton, R. P., Harvey, P. H. \& Pagel, M. Phylogenetic analysis and 408 comparative data: A test and review of evidence. Am Nat 160, 712-726, 409 doi:10.1086/343873 (2002). 


\section{Acknowledgements}

412 We thank Ciara O'Donovan, Mark Pagel, Graeme Ruxton, and Manabu Sakamoto

413 for helpful discussions during the course of this research. JB is funded by the 414 Leverhulme Trust (ECF-017-22). CV was funded by a Leverhulme Trust Research 415 Project Grant (RPG-2017-071).

\section{Author Contributions}

417 All authors contributed to all aspects of this research.

\section{Author Information}

$419{ }^{1}$ School of Biological Sciences, University of Reading, Reading RG6 6BX, United 420 Kingdom.

4212 School of Earth Sciences, University of Bristol, Life Sciences Building, Tyndall 422 Avenue, Bristol BS8 1TQ, United Kingdom.

$423{ }^{3}$ School of Life Sciences, University of Lincoln, Joseph Banks Laboratories, Green 424 Lane, Lincoln LN6 7DL, United Kingdom.

425

*Correspondence to c.d.venditti@reading.ac.uk and shumphries@lincoln.ac.uk.

428 The authors declare no competing financial interests.

\section{Data Availability Statement}

430 The phylogeny we generated in this study (presented in Figure 2a) is included as 431 Supplementary Information (Supplementary Data File 1) with this article. No other 432 data was generated or analysed during the current study. All data used are available 433 from published sources and are cited in the main text where appropriate. 


\section{Software and Code}

435 All analyses in this research were conducted using readily available, published

436 programs and are cited in the text. Version numbers of the programs we used are as

437 follows: Tracer v1.6. (2015), MATLAB v9.2 (2017), R v3.4.4 (2017), BEAST 2 (2014), 438 BayesTraits v3 (2018). 